\title{
Perceptual expertise enhances the resolution but not the number of representations in working memory
}

\author{
Miranda Scolari, EdWard K. Vogel, ANd EdWARd AWH \\ University of Oregon, Eugene, Oregon
}

\begin{abstract}
Despite its central role in cognition, capacity in visual working memory is restricted to about three or four items. Curby and Gauthier (2007) examined whether perceptual expertise can help to overcome this limit by enabling more efficient coding of visual information. In line with this, they observed higher capacity estimates for upright than for inverted faces, suggesting that perceptual expertise enhances visual working memory. In the present work, we examined whether the improved capacity estimates for upright faces indicates an increased number of "slots" in working memory, or improved resolution within the existing slots. Our results suggest that perceptual expertise enhances the resolution but not the number of representations that can be held in working memory. These results clarify the effects of perceptual expertise in working memory and support recent suggestions that number and resolution represent distinct facets of working memory ability.
\end{abstract}

Working memory enables the maintenance of a limited number of representations in a rapidly accessible and easily manipulated state. This form of memory storage is widely acknowledged as a core component of most complex cognitive activities (e.g., ACT-R, Anderson, 1993; EPIC, Meyer \& Kieras, 1997). Moreover, individual differences in working memory capacity are correlated with a variety of measures of general intelligence (Cowan et al., 2005). Thus, there has been widespread interest in understanding the factors that determine working memory capacity.

The present work focuses on how capacity in visual working memory is influenced by perceptual expertise. Perceptual expertise can be defined as an enhanced ability to carry out subordinate-level discriminations, typically as a result of extensive experience with a specific category of stimuli (e.g., Gauthier \& Tarr, 2002; Tanaka \& Taylor, 1991). It has been suggested that perceptual expertise enables more efficient mental representations of information (e.g., Gobet et al., 2001; Humphreys, Hodsoll, \& Campbell, 2005; Moore, Cohen, \& Ranganath, 2006), perhaps through "holistic" encoding that takes into account the relationships between individual features rather than just the features themselves. This raises the intriguing possibility that such efficient representations occupy less "space" in visual working memory, such that a greater number of objects can be stored. Curby and Gauthier (2007) examined this hypothesis by measuring change detection performance for faces, a class of stimuli for which the typical observer has perceptual expertise. In this paradigm (e.g., Luck \& Vogel, 1997; Pashler, 1988), subjects are asked to remember an array of items over a brief delay and then to indicate whether any of these items has changed in a subsequent test display. The number of objects that can be simultaneously maintained is estimated by measuring change detection accuracy as a function of the number items in the sample array (Cowan, 2001; Pashler, 1988). Curby and Gauthier found that when subjects were given adequate time to encode these complex stimuli, capacity estimates were significantly larger for upright faces than for inverted faces. In addition, Curby and Gauthier found that the influence of orientation was larger for faces than for cars, suggesting that the advantage for upright faces may have resulted from holistic encoding processes that are typically associated with faces but not with cars. Thus, it was suggested that holistic processing enabled the formation of more efficient memory representations, thereby allowing a larger number of objects to be held in working memory.

Curby and Gauthier (2007) also pointed out, however, that holistic processes might have enabled an increased number of features to be encoded into each of the object representations stored in memory. In this case, change detection performance may have been enhanced for upright relative to inverted faces even though the same number of each stimulus type was stored. That is, more detailed memory representations of the upright faces could have enabled better change detection for these stimuli than for the inverted versions of the same objects. Thus, at least two hypotheses might explain the benefits of holistic encoding on the maintenance of information in working memory. Holistic encoding may have allowed for more efficient representations of upright faces so that a larger number of faces could fit in visual

E.Awh, awh@uoregon.edu 
working memory. Alternatively, the same number of upright faces may have been encoded, but at a higher resolution than for the inverted faces. In this case, change detection could have been better for upright faces even though the same number of inverted faces had been represented in working memory. We attempted to discriminate between these alternatives using a procedure that provides separate estimates of the number and the resolution of the representations in working memory (Awh, Barton, \& Vogel, 2007).

The hypothesis that more efficient codes can enable the storage of a greater number of objects is relevant not only for our understanding of perceptual expertise and capacity, but also for the basic issue of how information load relates to capacity. In particular, there has been debate regarding whether the number of representations that can be maintained in working memory is determined by the overall complexity or information load of each object that is stored. For example, Luck and Vogel (1997) found that capacity estimates for objects made up of a single feature (i.e., color or orientation) were equivalent to those for multifeatured objects (e.g., colored lines of varying orientations). They concluded that capacity in visual working memory is determined by the total number of objects that must be maintained rather than by the total number of features (see also Vogel, Woodman, \& Luck, 2001). By contrast, Alvarez and Cavanagh (2004) demonstrated that change detection performance declined rapidly as "information load" or complexity increased across a broader range of object types. At first glance, these studies seem to support very different conclusions about the relationship between object complexity and the number of items that can be maintained in visual working memory.

Recently, Awh et al. (2007) reported evidence that may help to reconcile these results. Specifically, they examined whether the lower capacity estimates for complex objects may have been due to a concurrent increase in similarity between items in the complex categories. Such increases in interitem similarity could lead to reductions in change detection performance because of an increased probability of comparison errors during the final stage of the task. That is, change detection performance depends not only on the number of representations that can be maintained in working memory, but also on the observers' ability to discriminate between these stored representations and the new items that are presented during "change" trials. If interitem similarity is high, then changes can be missed in this procedure even though the changed item was stored in memory.

In line with this hypothesis, Awh et al. (2007) found that capacity estimates across four object categories that spanned the range of complexity tested by Alvarez and Cavanagh (2004) were strongly correlated $(r=.97)$ with a direct measure of interitem similarity within each category. Thus, comparison errors might be more likely for complex objects because of smaller differences between sample and test items during change trials. Awh et al. provided more direct support for this hypothesis using a modified change detection procedure in which each trial contained a randomly selected mixture of Chinese characters and shaded cubes, two object types with relatively high information load (as defined by Alvarez \& Cavanagh,
2004). In this procedure, half of the changes involved changes from cubes to characters or vice versa (crosscategory changes). The remaining half of the changes were within-category changes between cubes and cubes or between characters and characters. The rationale was that the relatively large changes in the cross-category-change condition would minimize comparison errors and enable more accurate estimates of the number of items represented. The results from the within-category-change trials replicated the findings of Alvarez and Cavanagh. There were monotonic reductions in capacity estimates as object complexity increased. However, the results from the cross-category-change trials showed that when comparison errors were minimized, equivalent capacity estimates were obtained for complex and simple objects. These data suggest that visual working memory represents a fixed number of items, regardless of object complexity.

The results of Awh et al. (2007) do not contradict the observation that change detection performance is strongly influenced by object complexity (Alvarez \& Cavanagh, 2004). However, it may be that the relationship between object complexity and change detection has more to do with the resolution than with the number of representations that can be stored in memory. Specifically, even though the results of Awh et al. suggest that an equivalent number of items can be maintained when object complexity is high, representations of higher resolution are required to detect changes between these highly similar items. ${ }^{1}$ The goal in the present study is to use the same approach to determine whether perceptual expertise facilitates change detection (Curby \& Gauthier, 2007) by increasing the number or the resolution of the representations that can be stored in working memory.

As did Awh et al. (2007), we measured change detection performance during both cross-category-change and within-category-change trials by using memory arrays that contained a mixture of faces and shaded cubes (the stimuli associated with the highest information load in the study by Alvarez \& Cavanagh, 2004). Thus, crosscategory changes involved changes from faces to cubes (or vice versa). If perceptual expertise allows for the storage of a larger number of upright than inverted faces in working memory, then the advantage for upright faces should be maintained even when observers are required to detect relatively large cross-category changes. In other words, if fewer inverted faces can be represented in memory, then even large changes will be missed for items that are not represented. The same reasoning accounts for observers' failure to detect salient color changes or large changes in visual scenes when mnemonic or attentional capacity has been exceeded (Luck \& Vogel, 1997; Simons, 1996; Simons \& Levin, 1997). By contrast, if perceptual expertise influences the resolution rather than the number of stored representations, then equivalent capacity estimates should be obtained for upright and inverted faces when sample-test similarity is low and comparison errors are minimized. Finally, we also obtained capacity estimates for simple colors so that we could reexamine previous assertions that a larger number of these simple objects can be stored in comparison with complex stimuli such as faces and cubes. 


\section{METHOD}

\section{Subjects}

Twelve people from the University of Oregon community participated in the experiment for either partial course credit or money for the 1-h session. Subjects had normal or corrected-to-normal vision.

\section{Stimuli}

The stimuli (illustrated in Figure 1) included shaded cubes (adapted from Alvarez \& Cavanagh, 2004), upright and inverted faces (adapted from the image database maintained by Libor Spacek, available at cswww.essex.ac.uk/mv/allfaces/index.html), and colored ovals. Each trial consisted of six objects presented in a circle around the fixation point, with a distance of approximately $3.2^{\circ}$ of visual angle between fixation and each object. The faces and colored ovals measured $3.7 \mathrm{~cm}$ in height (subtending approximately $4.6^{\circ}$ ) and $2.9 \mathrm{~cm}$ in width (subtending approximately $3.6^{\circ}$ ), and the shaded cubes measured $2.9 \mathrm{~cm}$ in both height and width (subtending approximately $3.6^{\circ}$ ). Trials consisted of either faces and shaded cubes (face/cube trials) or colored ovals (color trials). The colors used included six easily discriminated hues (red, green, blue, yellow, black, and cyan). The test screen included a single probe item in a location previously occupied by one of the items in the memory array. In same trials, one item previously presented in the memory array appeared alone in the same location. If a change occurred on a face/cube trial, test items were either from the same category as the memory item (within-category change) or from the other category (cross-category change). For color trials, a colored oval always changed into another colored oval.

\section{Procedure}

All subjects participated in two parts of the experiment that involved four blocks of 40 trials each. Both parts included trials that employed within-category and cross-category changes of faces and cubes ( $80 \%$ of trials), as well as trials that tested memory for simple colors ( $20 \%$ of trials). One part used upright faces, whereas the other used inverted faces. The order of these conditions was counterbalanced across subjects. Figure 2 illustrates the major components of each trial. Each trial started with the onset of a black fixation dot in the center of the screen. One hundred ninety-two milliseconds following fixation, the sample array appeared; face/cube trials remained on screen for $1,000 \mathrm{msec}$ (to ensure adequate encoding time for orientation effects to emerge; see Curby \& Gauthier, 2007) and color trials remained on screen for $500 \mathrm{msec}$. The sample array was followed by a 1,000-msec delay period, after which the test object appeared and remained on screen until response. Half of all trials were change trials. Five of the 11 changes in the face/cube condition involved small, within-category changes, and the remaining 6 trials involved big, cross-category changes. Subjects pressed a key to indicate whether the test item matched ("z" key) or mismatched ("/" key) the sample item that had previously occupied the same location.

\section{RESULTS AND DISCUSSION}

Working memory capacity estimates were calculated using the formula developed by Pashler (1988) and refined by Cowan (2001). ${ }^{2}$ A one-way ANOVA of the data from the within-category and color change trials revealed

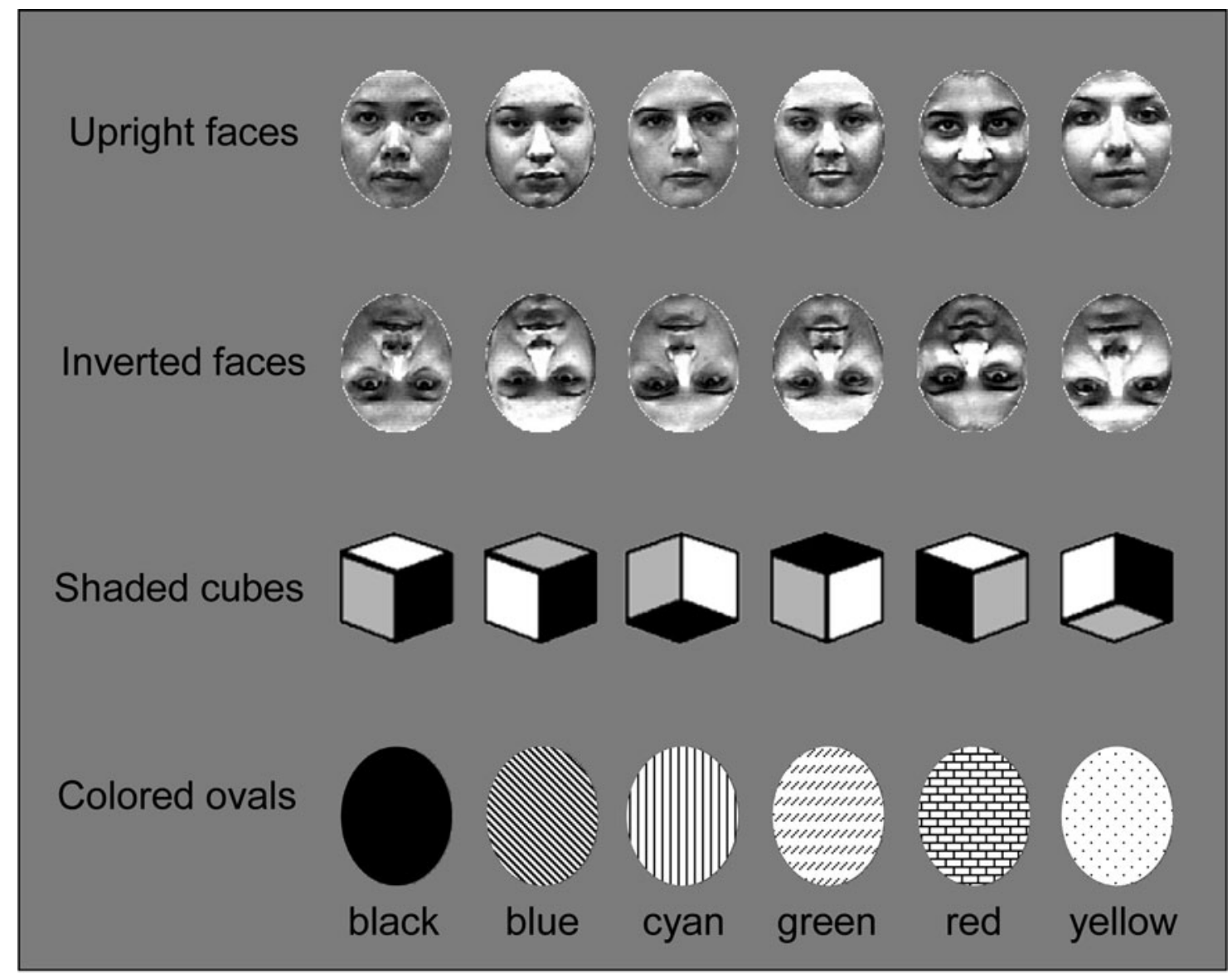

Figure 1. All possible objects from each of the categories. The shaded cubes were adapted from Alvarez and Cavanagh (2004), and the faces were adapted from an image database maintained by Libor Spacek. 

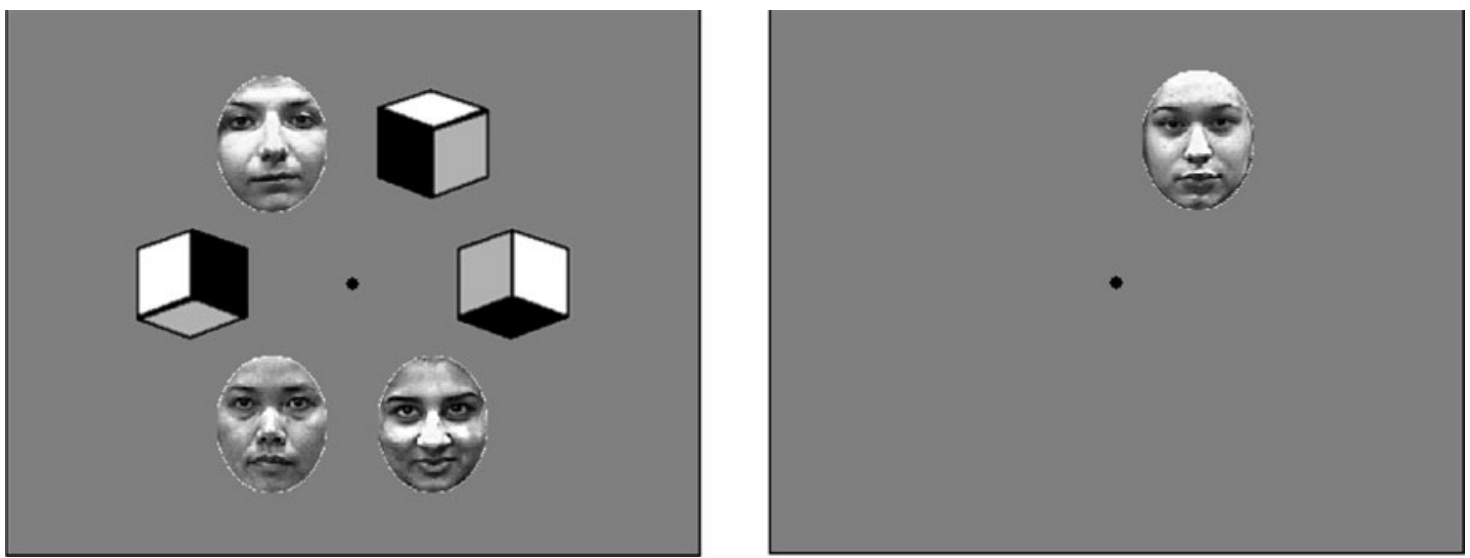

Figure 2. The left panel shows a face/cube memory array from the upright face condition, and the right panel shows the single probe. This trial is a cross-category change, in which a shaded cube has changed into an upright face.

significant differences in the capacity estimates for simple colors, cubes, upright faces, and inverted faces $[F(3,40)=$ $12.09, p<.001]$. The pattern of effects in the withincategory change trials (i.e., "small change" trials, illustrated in Figure 3) replicated the observations of both Alvarez and Cavanagh (2004) and Curby and Gauthier (2007). Planned contrasts revealed higher capacity estimates for color changes $(k=3.53)$ than for cube changes $(k=1.15)$ $[t(40)=4.50, p<.001]$, and estimates were higher for upright faces $(k=1.72)$ than for inverted faces $(k=0.51)$ $[t(40)=2.28, p=.028]$. This replication of previous findings indicates that our procedure was sensitive to the key effect of orientation on face change detection, as well as the advantage for the detection of changes between simple colors over detection of changes between items of more complex object categories, such as faces and cubes.

The key question in this experiment was whether the advantage for upright over inverted faces would be main- tained even when comparison errors were minimized during the cross-category change trials. If visual expertise influences working memory via an increase in the number of stored representations, then capacity estimates should have been greater for upright than for inverted faces even during the cross-category-change trials when the changes were big. However, if expertise enables the same number of representations to be maintained with higher resolution, then capacity estimates should be equivalent for upright and inverted faces when the possibility of comparison errors was minimized. The latter hypothesis was supported. Capacity estimates for upright $(k=4.11)$ and inverted $(k=4.01)$ faces were equivalent during the cross-category change trials when faces changed into cubes $[t(10)=0.31, p=$ .76], despite the fact that small, within-category changes were detected almost three times more frequently for upright than for inverted faces. Thus, although we replicated Curby and Gauthier's (2007) finding that change detection

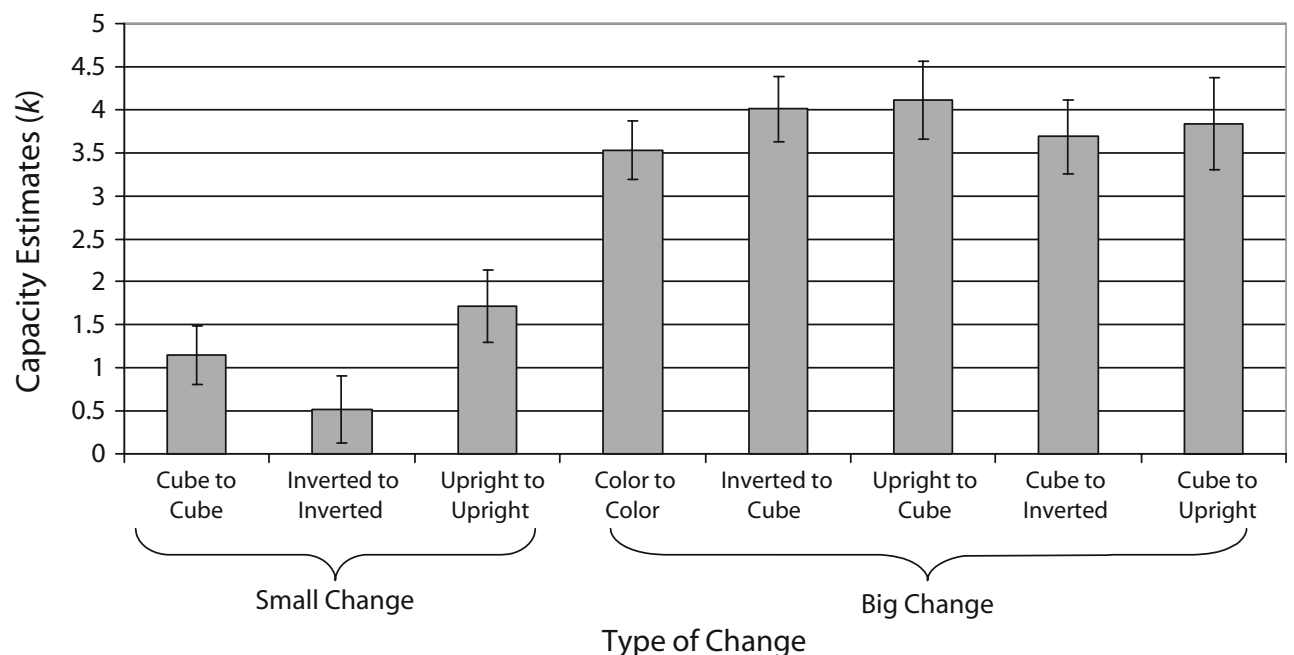

Figure 3. Working memory capacity $(k)$ estimates for different change conditions, depending on whether changes were small or big. All small changes were from the within-category condition. All big changes were from the cross-category-change condition except for the color condition, in which interitem similarity was also very low. 
accuracy is higher for upright than for inverted faces, the benefits of upright presentations may not indicate a difference in the number of upright and inverted faces that can be stored in memory. Instead, we suggest that perceptual expertise enables upright faces to be represented in working memory with higher resolution than inverted faces.

These results also suggest an alternative explanation of the apparent differences in capacity that have been previously reported among faces, cubes, and simple colors (Alvarez \& Cavanagh, 2004; Eng, Chen, \& Jiang, 2005). Pairwise $t$ tests revealed equivalent capacity estimates in the color condition and all of the cross-category-change conditions [upright face to cube, $k=4.11, t(10)=1.51, p=.16$; inverted face to cube, $k=4.01, t(10)=1.32, p=.22$; cube to upright face, $k=3.84, t(10)=0.83, p=.42$; cube to inverted face, $k=3.68, t(10)=0.98, p=.35]$. Thus, when comparison errors were minimized, capacity estimates for the least complex objects we tested (i.e., simple colors) were equivalent to those for shaded cubes, upright faces, and inverted faces. These data replicated the findings of Awh et al. (2007) and provide further support for the claim that object complexity influences the resolution but not the number of items that can be represented in working memory.

We considered whether the presence of cross-category changes may have elicited a "low-resolution" strategy in this experiment, in which observers encoded only whether the stimuli fell into the face category or the cube category. Could subjects have attained higher capacity estimates by refraining from encoding the details of the complex objects? Two results suggest otherwise. First, the capacity estimates that we obtained for the shaded cubes $(K=$ 1.15 ) were in line with previous studies that measured performance with virtually identical stimuli but without any cross-category changes (Alvarez \& Cavanagh, 2004; Awh et al., 2007, Experiment 1; Eng et al., 2005). This suggests that subjects in the present experiment encoded more than category-level information for these stimuli. Second, the significant advantage that we observed for upright relative to inverted faces in the within-category condition suggests that enough detail was encoded to provide a test bed for understanding the effects of perceptual expertise.

The results from the cross-category change trials suggest that an equivalent number of items can be stored in working memory regardless of the complexity of the items or the benefits of perceptual expertise. Nevertheless, the results from the within-category trials do show clear effects of complexity and perceptual expertise. This apparent discrepancy is consistent with our hypothesis that there is a shift in the limiting factor for change detection when sample-test similarity increases (Awh et al., 2007). When sample-test similarity is low (i.e., in the cross-category-change and color trials), change detection is limited by the number of items that can be simultaneously represented in working memory. But when sample-test similarity increases (i.e., between the face and cube stimuli), performance is limited instead by the probability of comparison errors. In this case, errors in detecting changes in complex objects may provide a measure of the effective resolution of the representations in memory. This hypothesis can account for why capacity estimates were equivalent among simple colors, cubes, and faces when comparison errors were minimized in the crosscategory change condition.

This two-factor model of working memory can also be tested by examining the pattern of individual differences within these tasks. If the conditions with large changes enable a relatively pure estimate of the number of representations that can be stored in working memory, then performance should be strongly correlated across all of these conditions (face-to-cube changes, cube-to-face changes, and color changes). However, if subjects' ability to detect small changes (i.e., face-to-face changes or cube-to-cube changes) reflects a different aspect of memory ability such as resolution, then there may be little or no relationship between an individual's abilities to detect small changes and large changes. These predictions contrast with those of models that assert a direct trade-off between number and complexity in visual working memory. For example, past findings that change detection is impaired as object complexity increases (Alvarez \& Cavanagh, 2004; Eng et al., 2005) have led to the hypothesis that complex objects occupy more "space" within a limited buffer, so that capacity limits are reached after fewer objects have been stored. If this were the case, then individuals with a large capacity for simple objects should also have a relatively large capacity for complex objects, leading to strong correlations between change detection scores with simple and complex stimuli.

We tested these predictions by examining the correlations between capacity estimates in conditions in which sample-test similarity was high or low (see Table 1 for a full list of pairwise correlations; see Figure 4 for a repre-

Table 1

Correlations Between Capacity Estimates in Each of the Conditions

\begin{tabular}{|c|c|c|c|c|c|c|c|c|}
\hline$r$ Values $(n=11)$ & Cube & $\begin{array}{c}\text { Upright } \\
\text { Face }\end{array}$ & $\begin{array}{c}\text { Inverted } \\
\text { Face }\end{array}$ & Color & $\begin{array}{c}\text { Cube to } \\
\text { Upright } \\
\text { Face }\end{array}$ & $\begin{array}{c}\text { Cube to } \\
\text { Inverted } \\
\text { Face }\end{array}$ & $\begin{array}{l}\text { Upright } \\
\text { Face } \\
\text { to Cube }\end{array}$ & $\begin{array}{c}\text { Inverted } \\
\text { Face } \\
\text { to Cube }\end{array}$ \\
\hline \multicolumn{9}{|l|}{ Cube } \\
\hline Upright face & .12 & & & & & & & \\
\hline Inverted face & .43 & .62 & & & & & & \\
\hline Color & .004 & -.05 & .18 & & & & & \\
\hline Cube to upright face & -.15 & -.18 & .31 & .72 & & & & \\
\hline Cube to inverted face & -.12 & .01 & .27 & .94 & .80 & & & \\
\hline Upright face to cube & -.07 & -.18 & .08 & $.69^{*}$ & .84 & .59 & & \\
\hline Inverted face to cube & -.08 & -.37 & -.02 & $.78^{*}$ & .65 & .46 & .66 & \\
\hline Face/cube & -.12 & -.20 & .20 & .78 & .96 & .83 & .89 & .78 \\
\hline
\end{tabular}

Note-Bold values denote significance at $p<.05 . \quad{ }^{*}$ Correlations in which one outlier was removed. 

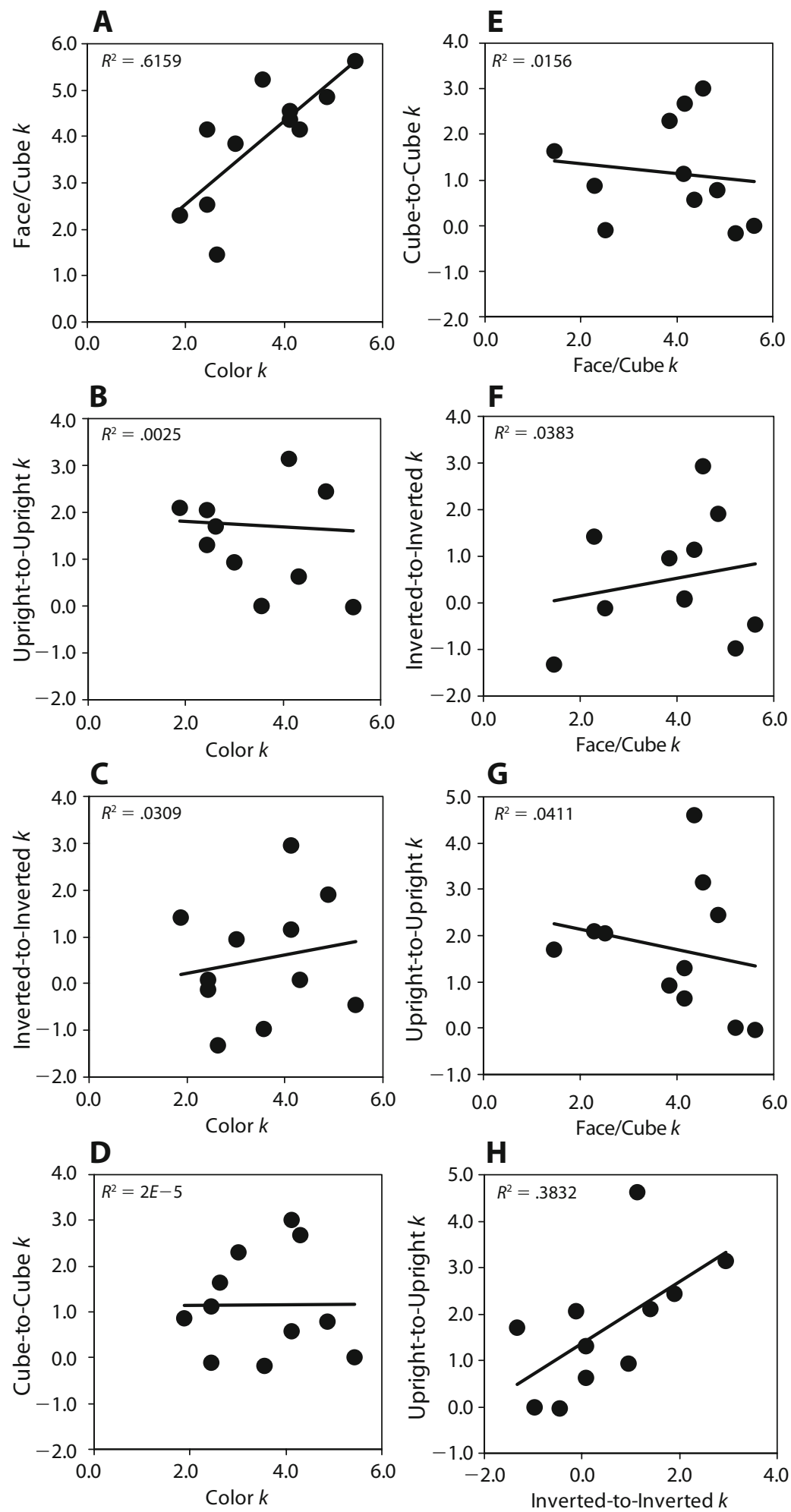

Figure 4. Correlations between individual capacity estimates in a representative sample of the conditions. See Table 1 for a full correlation matrix.

sentative set of scatterplots). Collapsing across the crosscategory change conditions (i.e., face-to-cube and cube-toface changes), we found strong correlations between the accuracy of change detection in the cross-category-change condition and that in the simple color condition $(r=.78$, $p=.008$; see panel A of Figure 4). Given that the simple color condition provides a reasonable benchmark for the maximum capacity of working memory (Alvarez \& Ca- 
vanagh, 2004), these data suggest that the cross-categorychange condition measures capacity in the same online memory system. Because comparison errors are minimal in these conditions, and because previous studies have suggested that encoding limits do not strongly determine performance with stimuli of this type (e.g., Alvarez \& Cavanagh, 2004; Luck \& Vogel, 1997), our hypothesis is that these conditions measure a common limit for the number of items that can be held in working memory. By contrast, there was no apparent relationship between performance in the simple color condition and performance in the within-category-change conditions, in which sample-test similarity was high (upright face: $r=-.05$; inverted face: $r=.18$; cube: $r=.004$; see panels $\mathrm{B}, \mathrm{C}$, and D of Figure 4). Furthermore, there were no correlations between performance in the cross-category-change condition (i.e., cube/face and face/cube changes) and the within-categorychange conditions (cube: $r=-.12$; inverted face: $r=.20$; upright face: $r=-.20$; see panels $\mathrm{E}, \mathrm{F}$, and $\mathrm{G}$ of Figure 4). A test of differences between within-sample correlations revealed that the correlation between capacity estimate in the color condition and in the cross-category-change conditions was significantly larger than that between capacity estimates in the color condition and the within-categorychange conditions (all $z \mathrm{~s}>1.96$ ). These data replicate the findings of Awh et al. (2007) and support our hypothesis that merely changing the stimuli that are presented in a change detection procedure can cause a qualitative shift in the ability that is measured. Moreover, these results cast doubt on models of working memory that posit a single pool of resources to explain limitations in the number and resolution of representations in working memory. Instead, these data suggest that number and resolution are relatively distinct aspects of working memory ability.

Finally, although it is striking that we found no correlation between capacity estimates with simple colors and those with upright and inverted faces, there might be concern that the estimates with upright and inverted face stimuli may not have been reliable enough to provide a strong test of this relationship. This concern is addressed, however, by the finding that there was a significant correlation between performance in the within-category-change condition with upright faces and inverted faces $(r=.62$, $p=.04$; see panel $\mathrm{H}$ of Figure 4). Thus, these conditions reveal stable individual differences in the detection of face changes, suggesting that they provide a reasonable test of whether this ability is correlated with the number of items that an individual can maintain in working memory.

\section{CONCLUSIONS}

Using a change detection procedure, Curby and Gauthier (2007) demonstrated a clear advantage for upright over inverted faces. Given that the discrimination of upright faces is one of the best-documented examples of expert perception, these data provide compelling evidence that perceptual expertise enhances visual working memory. Nevertheless, questions remained regarding the precise nature of this enhancement, because differences in the accuracy of change detection could be explained by differences in the number or the resolution of the representations in memory. In the present work, we attempted to resolve this issue by using a procedure that enabled separate estimates of the number and resolution of the representations in working memory.

Our results replicated those of Curby and Gauthier (2007) by showing that change detection was superior for upright than for inverted faces. However, this advantage for upright faces was eliminated when sample-test similarity was reduced and the probability of comparison errors was minimized. Thus, we conclude that the same number of items is maintained in visual working memory regardless of contributions from perceptual expertise. Our hypothesis is that perceptual expertise influences the effective resolution rather than the number of representations in working memory. From this perspective, higher resolution representations of the upright faces led to a reduced probability of comparison errors and higher accuracy in the change detection procedure.

Our primary conclusions rest on the assumption that the cross-category-change condition and the color condition measure a common aspect of memory ability. Because both of these conditions minimize errors during the comparison stage of the task, we suggest that they provide a relatively pure estimate of the number of items that can be simultaneously maintained in visual working memory. However, we also considered the possibility that category knowledge might allow faces and cubes to be distinguished in a qualitatively different way than that in which the individual colors were distinguished in this study. Given that categorical information can be extracted relatively quickly, would this provide an "unfair" advantage in the cross-category-change condition? The present study cannot rule out the possibility that categorical coding provides an advantage for detecting the changes between faces and cubes. It is also plausible, however, that categorical perception aids performance in the simple color condition. Thus, although interesting questions remain regarding the role of category knowledge in change detection (e.g., Olsson \& Poom, 2005), we maintain that these conditions provide a valid estimate of the number of complex items that can be held in memory. The strong correlation between the color and the cross-category-change conditions supports this point by showing that performance in the cross-category-change condition was likely to be limited by the same core ability as in the color condition.

Our hypothesis regarding improved resolution for upright faces is consistent with previous studies that have documented enhanced discrimination of upright in comparison with inverted faces (Yin, 1969), including both configural and component aspects of these stimuli (Rhodes, Hayward, \& Winkler, 2006). However, because these studies have been primarily interested in measuring the acuity of face processing, the number of stimuli to be apprehended is typically not the limiting factor for discrimination. Thus, the present results address a relatively new question with regard to how perceptual expertise interacts with the number of stimuli that can be simultaneously processed. Our results suggest that higher acuity perceptual representations do not necessarily occupy less 
"space" in working memory, to the extent that the upper limit on the number of represented items is not affected by perceptual expertise. A more productive perspective may be one that focuses instead on the psychological "distance" between representations (see, e.g., Valentine, 1991, for a discussion of "face space"), such that the spacing between representations is wider for upright than for inverted faces. This notion of representational space dovetails with our emphasis on the influence of sample-test similarity in the change detection procedure.

Finally, these data are relevant to the broad issue of how complexity and capacity are related in visual working memory. In line with the results of Awh et al. (2007), we found that when comparison errors were minimized via cross-category changes, an equivalent number of items was represented in working memory regardless of the complexity of those items. Thus, although previous studies have shown that change detection performance declines as the complexity of the remembered items increases (Alvarez \& Cavanagh, 2004; Eng et al., 2005), these declines may have reflected changes in the resolution rather than in the number of the representations in memory. A correlational analysis also supported the hypothesized distinction between number and resolution. Although we found robust correlations between change detection scores in various conditions in which sample-test similarity was low and performance was limited by the number of items in working memory, none of these conditions predicted performance in conditions in which sample-test similarity was high and performance was limited by mnemonic resolution. Thus, we suggest that number and resolution may represent distinct facets of working memory ability.

\section{AUTHOR NOTE}

We are grateful to Libor Spacek for permitting us to use face images from his online database, and to Tara Sprague and Veronica Perez for assistance with the data collection. Correspondence concerning this article should be addressed to E. Awh, 1227 University, Eugene, OR 97405 (e-mail: awh@uoregon.edu).

\section{REFERENCES}

Alvarez, G. A., \& Cavanagh, P. (2004). The capacity of visual shortterm memory is set both by visual information load and number of objects. Psychological Science, 15, 106-111.

Anderson, J. R. (1993). Problem solving and learning. American Psychologist, 48, 35-44.

Awh, E., Barton, B., \& Vogel, E. (2007). Visual working memory represents a fixed number of items, regardless of complexity. Psychological Science, 18, 622-628.

Cowan, N. (2001). The magical number 4 in short-term memory: A reconsideration of mental storage capacity. Behavioral \& Brain Sciences, 24, 87-185.

Cowan, N., Elliott, E. M., Saults, J. S., Morey, C. C., Mattox, S. M., Hismuatullina, A., \& Conway, A. R. A. (2005). On the capacity of attention: Its estimation and its role in working memory and cognitive aptitudes. Cognitive Psychology, 51, 42-100.

Curby, K. M., \& Gauthier, I. (2007). A visual short-term memory advantage for faces. Psychonomic Bulletin \& Review, 14, 620-628.

ENG, H. Y., CHEN, D., \& JiAng, Y. (2005). Visual working memory for simple and complex stimuli. Psychonomic Bulletin \& Review, 12, 1127-1133.

GAUTHIER, I., \& TARR, M. J. (2002). Unraveling mechanisms for expert object recognition: Bridging brain activity and behavior. Journal of Experimental Psychology: Human Perception \& Performance, 28, 431-446.

Gobet, F., Lane, P. C., Croker, S., Cheng, P. C., Jones, G., Oliver, I., \& Pine, J. M. (2001). Chunking mechanisms in human learning. Trends in Cognitive Sciences, 5, 236-243.

Humphreys, G. W., Hodsoll, J., \& Campbell, C. (2005). Attending but not seeing: The "other race" effect in face and person perception studied through change blindness. Visual Cognition, 12, 249-262.

LuCK, S. J., \& Vogel, E. K. (1997). The capacity of visual working memory for features and conjunctions. Nature, 390, 279-281.

MeYer, D., \& Kieras, D. (1997). A computational theory of executive cognitive processes and multiple-task performance: Part 1. Basic mechanisms. Psychological Review, 104, 3-65.

Moore, C. D., Cohen, M. X., \& Ranganath, C. (2006). Neural mechanisms of expert skills in visual working memory. Journal of Neuroscience, 26, 11187-11196.

Olsson, H., \& Poom, L. (2005). Visual memory needs categories. Proceedings of the National Academy of Sciences, 102, 8776-8780.

PAshler, H. (1988). Familiarity and visual change detection. Perception \& Psychophysics, 44, 369-378.

Rhodes, G., Hayward, W. G., \& Winkler, C. (2006). Expert face coding: Configural and component coding of own-race and other-race faces. Psychonomic Bulletin \& Review, 13, 499-505.

Simons, D. J. (1996). In sight, out of mind: When object representations fail. Psychological Science, 7, 301-305.

Simons, D. J., \& Levin, D. T. (1997). Change blindness. Trends in Cognitive Sciences, 1, 261-267.

TANAKa, J. W., \& TAYloR, M. (1991). Object categories and expertise: Is the basic level in the eye of the beholder? Cognitive Psychology, 23, 457-482.

VAlEntine, T. (1991). A unified account of the effects of distinctiveness, inversion, and race in face recognition. Quarterly Journal of Experimental Psychology, 43A, 161-204.

Vogel, E. K., Woodman, J., \& LUCK., S. J. (2001). Storage of features, conjunctions and objects in visual working memory. Journal of Experimental Psychology: Human Perception \& Performance, 27, 92-114.

YIN, R. K. (1969). Looking at upside-down faces. Journal of Experimental Psychology, 81, 141-145.

\section{NOTES}

1. We are suggesting that comparison errors during change detection are caused by memory representations with limited resolution. However, our data do not rule out the alternative explanation that the errors are caused by a flawed comparison process that operates on otherwise veridical representations in memory. We use the term resolution for ease of exposition, even though this issue has not been fully resolved.

2. Capacity $(k)$ is estimated on the basis of the number of items in the display $(N)$, the hit rate for detecting changes (HR), and the false alarm rate (FA). $k=N *(\mathrm{HR}-\mathrm{FA})$

(Manuscript received February 23, 2007; revision accepted for publication June 13, 2007.) 